\title{
"Too Young to Have a Broken Heart": Spontaneous Coronary Artery Dissection Causing ST-Elevation Myocardial Infarction in a Young Adult: A Case Report
}

\author{
Marc Denver A. Tiongson a, b, Eric Oliver D. Sison ${ }^{\mathrm{a}}$, Jaime Alfonso M. Aherrera ${ }^{\mathrm{a}}$, \\ Dioscoro DC Bayani, II ${ }^{\text {a }}$, Joerelle Mojica ${ }^{\text {a }}$ Nashiba Daud ${ }^{\text {a }}$, \\ John Daniel Ramosa
}

\begin{abstract}
ST-elevation myocardial infarction (STEMI) rarely occurs among patients 18 to 34 years old. Spontaneous coronary artery dissection (SCAD) is a rare cause of STEMI and is frequently described among patients in peripartum period. SCAD has a high mortality rate if not recognized and treated immediately. We present a case of SCAD presenting as STEMI in a 19-year-old nonpregnant patient. A 19-yearold female with chronic kidney disease, complained of sudden onset substernal chest pain. Physical examination showed a blood pressure of 140/90 mm Hg, HR of $112 \mathrm{bpm}$, with note of rales, pedal edema, and cold clammy extremities. Electrocardiogram showed ST-elevation in leads V3 to V6. Cardiac troponin was elevated and echocardiography revealed left ventricular segmental hypokinesia and depressed systolic function. Patient was diagnosed with acute anterolateral wall STEMI. Coronary angiogram revealed total occlusion of the mid-segment of the left anterior descending artery (LAD), while the rest of the coronary arteries were strikingly normal. After initial balloon angioplasty and stenting of the mid LAD, coronary artery dissection was noted at the distal LAD. A stent was successfully deployed, achieving TIMI flow grade III with no residual stenosis. She remained stable and was discharged improved. STEMI rarely happens in the young adults. Moreover, literature highlights the rarity of STEMI caused by SCAD. SCAD usually occurs among young pregnant patients without risk factors for atherosclerosis. We highlighted the significance of suspecting SCAD among young patients who present with STEMI and prompt treatment with revascularization in clinical situations such as this case. SCAD remains to be a rare cause of STEMI. However, SCAD should be considered among young individuals with STEMI. Treatment is primarily medical unless there is persistent chest pain and/or ischemic ECG changes, hemodynamic instability, or unstable arrhythmia, where revascularization is necessary.
\end{abstract}

Manuscript submitted February 26, 2018, accepted April 30, 2018

aSection of Cardiology, Philippine General Hospital, University of the Philippines, Manila, Philippines

${ }^{b}$ Corresponding Author: Marc Denver A. Tiongson, Section of Cardiology, Philippine General Hospital, University of the Philippines, Manila, Philippines. Email: denver_tiongson@yahoo.com

doi: https://doi.org/10.14740/jmc3052e
Keywords: Spontaneous coronary artery dissection; Acute coronary syndrome; ST-elevation myocardial infarction

\section{Introduction}

ST-elevation myocardial infarction (STEMI) rarely occurs in the young adult. Among patients age 18 to 34 years old, STEMI happens in 5 of 1000 patients [1]. It is most commonly due to plaque rupture with underlying atherosclerosis [2]. However, in the young adult, especially women, other causes such as spontaneous coronary artery dissection (SCAD) can cause STEMI [3].

SCAD is a non-traumatic and non-iatrogenic separation of coronary artery walls [4]. It is a rare cause of myocardial infarction. Based on several registries, it occurs in 1 to 4 of 10,000 patients per year among patients suffering from acute coronary syndrome (ACS) $[5,6]$. Because of its low incidence, it is less recognized as the cause of STEMI. We reported a case of a 19-year-old patient with chronic kidney disease secondary to rapidly progressive glomerulonephritis who presented with STEMI as the presentation of SCAD.

\section{Case Report}

Our patient is a 19-year-old, female, Asian, who complained on sudden onset, substernal chest pain and left shoulder pain $14 \mathrm{~h}$ prior to admission. The pain was described as heaviness, substernal in location, moderate in intensity and associated with shortness of breath. The pain lasted for more than $3 \mathrm{~h}$ prompting initial consult at another institution.

The patient was diagnosed 1 year ago with chronic kidney disease secondary to rapidly progressive glomerulonephritis and has been on regular peritoneal dialysis then converted to hemodialysis for the past 9 months. She had prior intake of steroid for 3 months for her chronic glomerulonephritis. She also has hypertension for which she was taking amlodipine. She denies intake of any illegal drugs, smoking or alcohol. She denies use of contraceptive pills and claims to be not sexually active. Her father died of a massive heart attack at age 45 years 

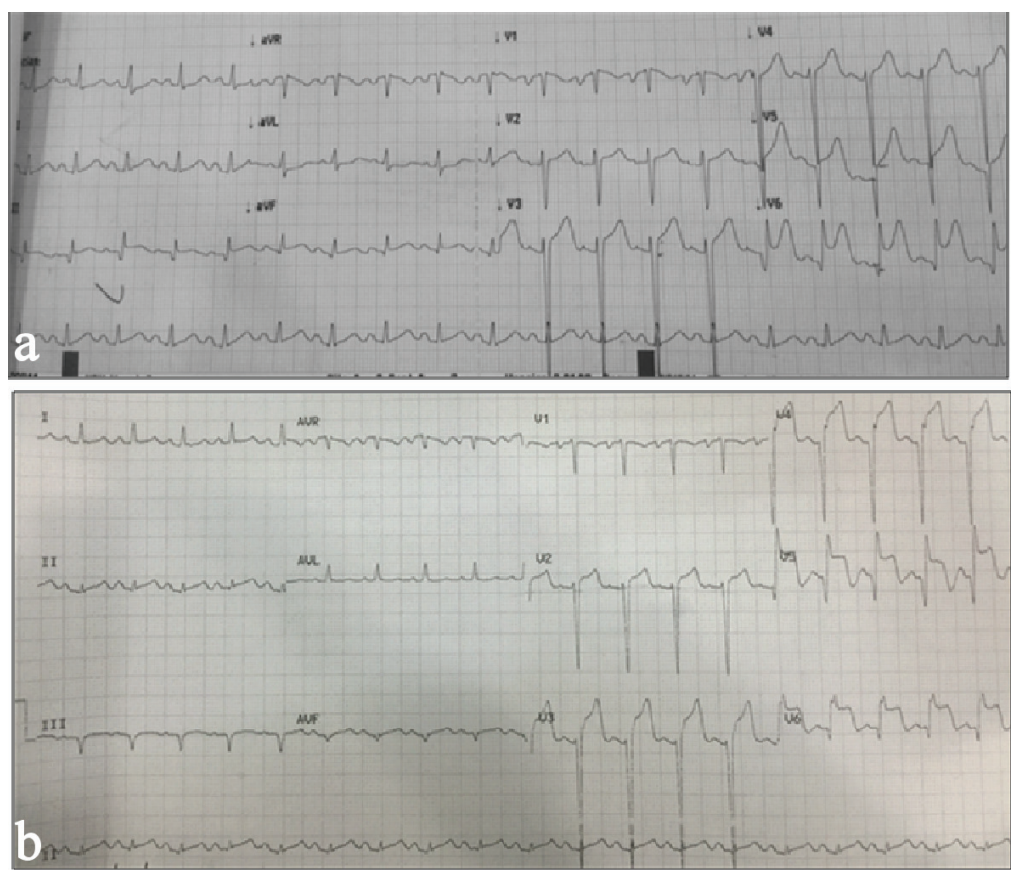

Figure 1. (a) Initial ECG showing regular sinus rhythm normal axis, anterolateral wall ST-elevation myocardial infarction. (b) Repeat ECG showing persistent ST-elevation myocardial infarction.

old.

Her initial ECG, which was taken 3 hours after the onset of chest pain, showed acute anterolateral wall ST elevation myocardial infarction (Fig. 1a). Initial troponin done at the other institution was $1.320 \mathrm{ng} / \mathrm{mL}$ (normal range $<0.35$ $\mathrm{ng} / \mathrm{mL}$ ). She was managed as a case of acute coronary syndrome. She was given with aspirin, clopidogrel, atorvastatin, enoxaparin, carvedilol, captopril, and isosorbide dinitrate. They then opted to transfer to our institution for further management.

Her physical examination at the emergency room (ER) showed a blood pressure (BP) of 140/90 mm Hg, HR of 112 bpm with note of rales, edema, good pulses and cold clammy extremities. Her ECG on admission showed up sloping ST elevation in leads V3 to V6 (Fig. 1b). Chest X-ray revealed multichamber cardiac enlargement, pulmonary congestive changes and IJ catheter. Repeat troponin I HS was 90,575 ng/L (normal range $<15.6 \mathrm{ng} / \mathrm{L}$ ). Her blood chemistry results were within acceptable limits saved for a creatinine of $508 \mathrm{mmol} / \mathrm{L}$.

Initial consideration was possible uremic pericarditis versus ACS. A two-dimensional echocardiogram was done showing a dilated left ventricle with segmental wall motion abnormality (hypokinesia entire anterior and septal wall, lateral wall at the apex and anteroseptal wall from base to apex) with EF of $17 \%$ (Simpsons). On Doppler studies, there was note of tricuspid and mitral regurgiation as well. ACS regimen was continued. She was also noted to have pulmonary congestion for which intravenous (IV) furosemide was given. There was reduction of the chest pain and improvement of her dyspnea.

Coronary angiogram was done on the second hospital day. Coronary angiogram showed no significant calcification. The left main, left circumflex, and the right coronary arteries were smooth and free of any coronary artery disease (Fig. 2). The left anterior descending artery (LAD) was totally occluded at the mid segment with some slow flow prior to the occlusion (Fig. 3a). We then proceeded with percutaneous coronary intervention (PCI) of the LAD where a 0.014 CHOICE PT PTCA wire was inserted up to the distal LAD. Multiple ballooning of the occluded segments using a $2.0 \times 20 \mathrm{~mm}$ balloon dilatation catheter did not restore flow. Deducing that the occlusion could most likely be due to a dissection with an intimal flap causing the obstruction, we proceeded to stent the mid LAD using a $2.5 \times 32 \mathrm{~mm}$ drug eluting stent (DES). This still failed to restore flow (Fig. 3b). We then used the stent balloon to dilate the distal LAD. This restored flow to the entire LAD and allowed us to visualize the coronary dissection in the distal LAD that caused the occlusion (Fig. 4a). We proceeded to stent the dissection with good result, achieving TIMI III flow with no residual stenosis (Fig. 4b). The patient remained stable, with no recurrence of chest pain. She was advised dual antiplatelet therapy with aspirin and clopidogrel for 1 year then aspirin indefinitely. All her other medications were continued. She was sent home on day 5 with no complications. Close monitoring of cardiac function through OPD follow-ups were encouraged.

\section{Discussion}

STEMI is very rare in the young adult population [1]. However, the etiology of MI in the young is usually due to causes other than coronary atherosclerosis. The more common mechanism for MI in the young adult would include the following: hypercoagulable state, vasculitides, embolic phenomenon and 

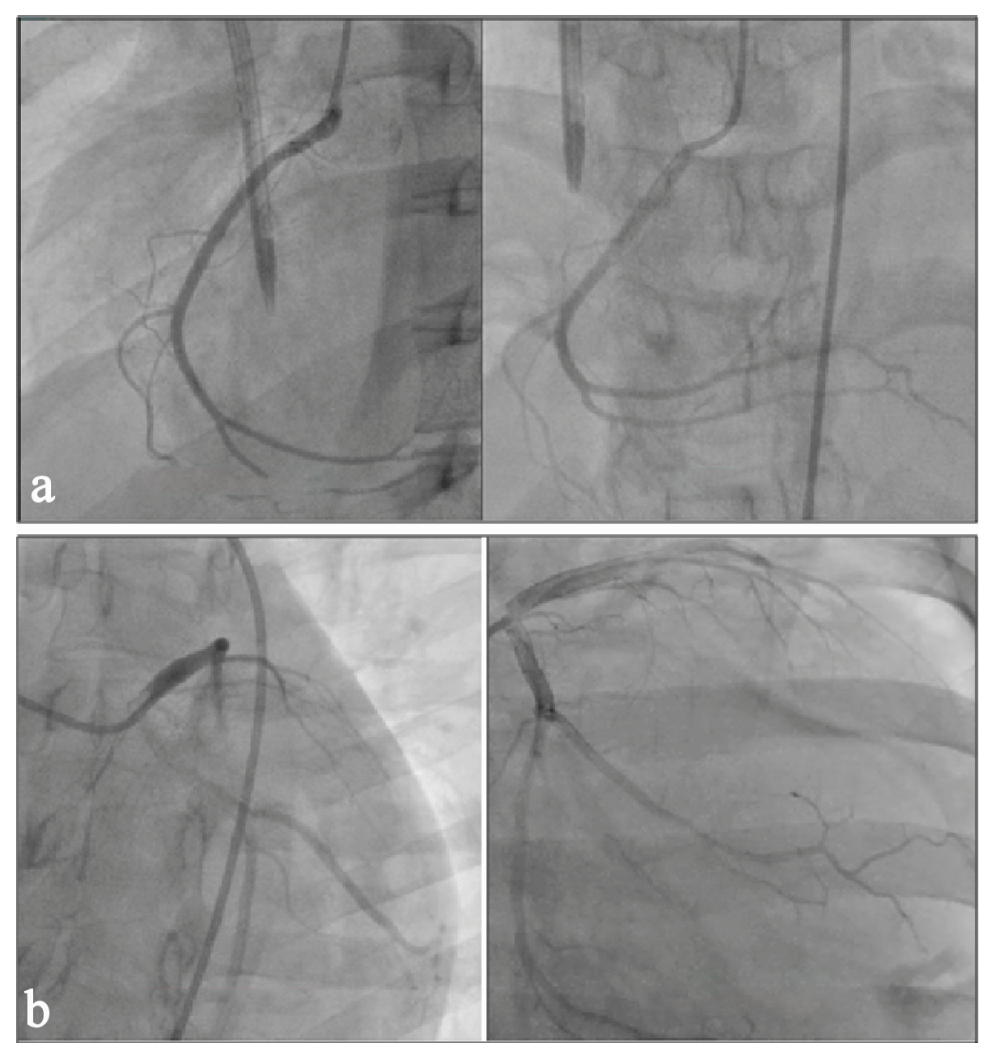

Figure 2. Right coronary angiogram (a) and left circumflex (b) angiogram showing good flow.

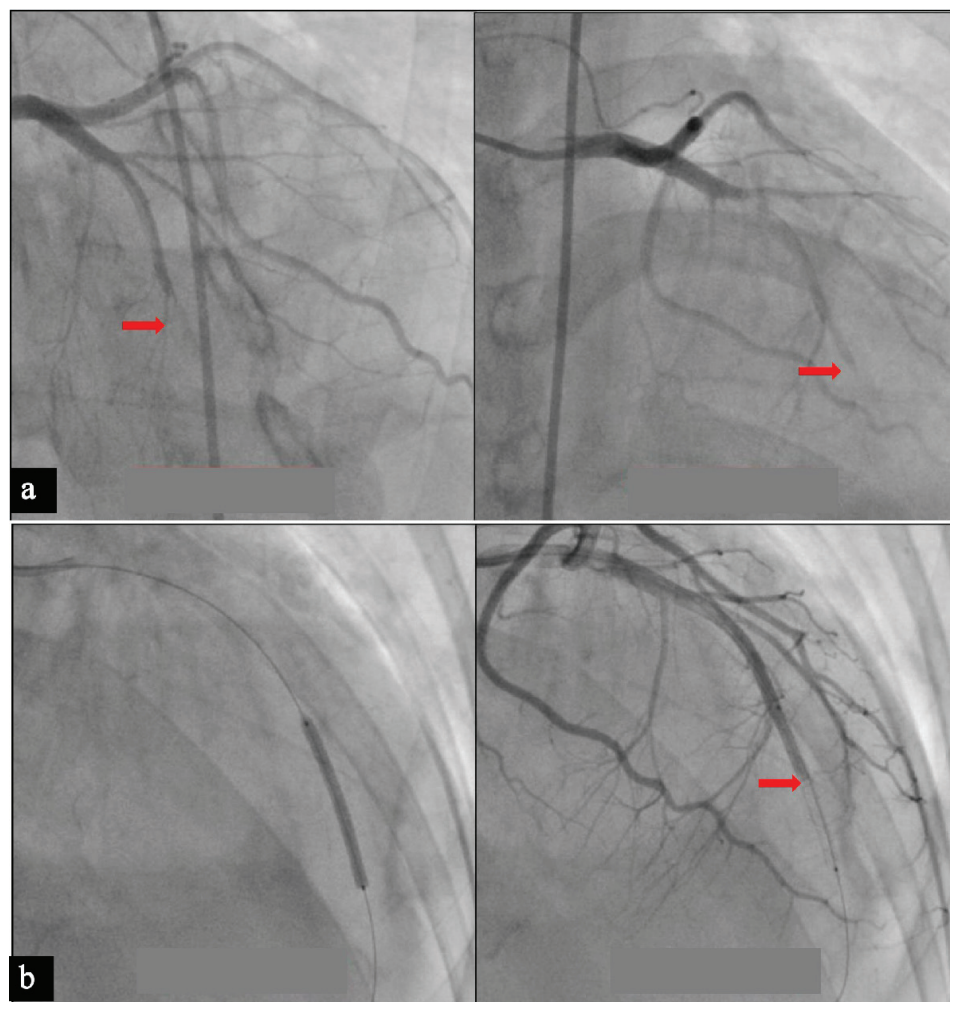

Figure 3. (a) Total occlusion at the mid left anterior descending artery. (b) Stenting of the mid left anterior descending artery (left) and angiogram showing failure to restore flow (right). 

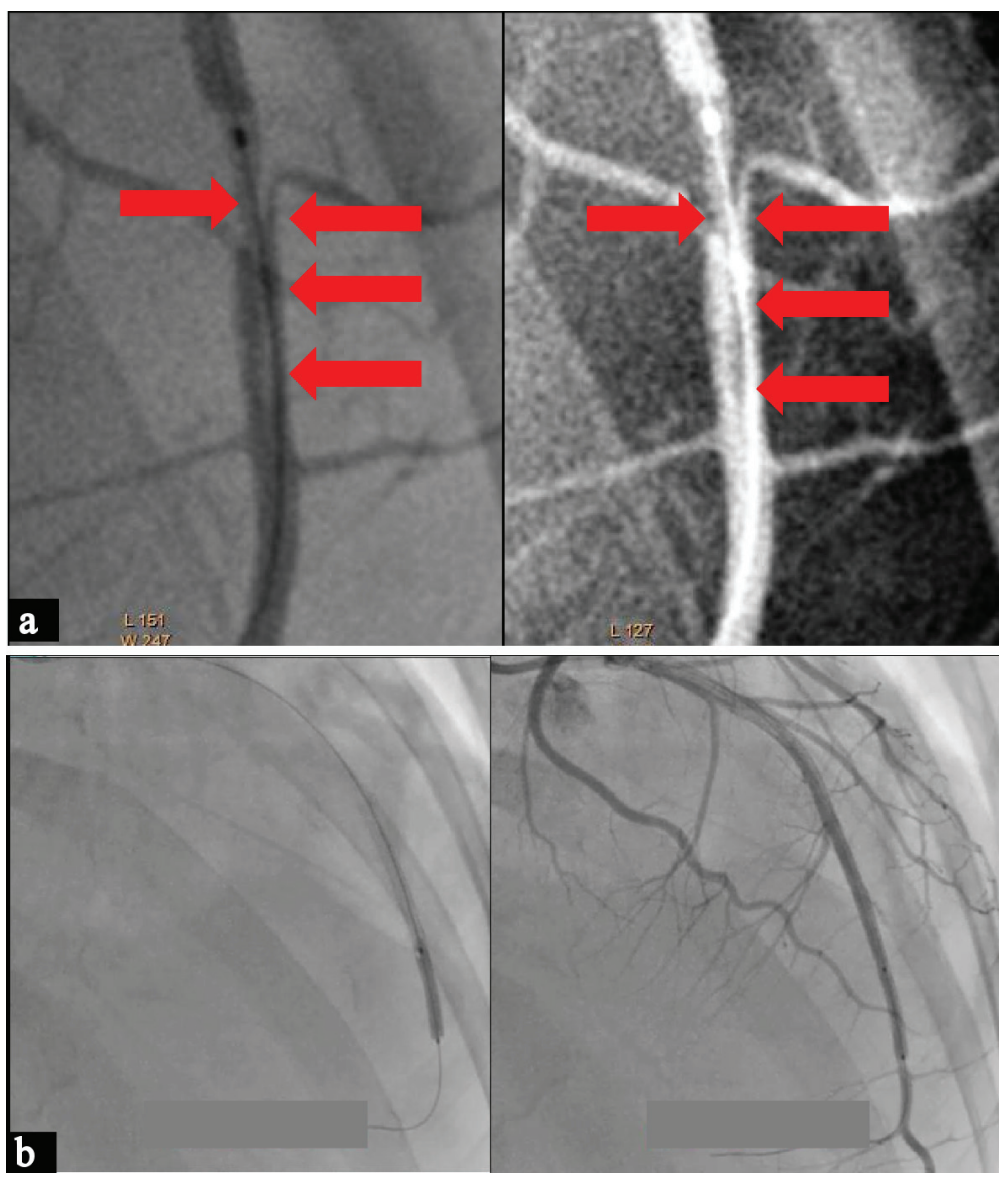

Figure 4. (a) Angiogram showing the LAD dissection (arrows),

(b) Stenting of the distal LAD and angiogram showing good flow with no residual stenosis.

spontaneous coronary artery dissection. In addition, an earlier study conducting autopsy among victims of homicides, suicides or vehicular crash, advanced coronary atheroma was only seen in patients in less than $2 \%$ [7].

In young females, often without traditional risk factors, SCAD can cause acute coronary syndrome, ranging in presentation from unstable angina, non-ST elevation myocardial infarction (NSTEMI), STEMI, sudden cardiac death or arrhythmia [8]. Therefore, SCAD should be recognized as one of the causes of ACS in the young.

\section{Epidemiology}

In one of the ACS registries of patients with STEMI, it was found out that SCAD occurs in $0.4 \%$ to $0.5 \%$ of the population [5]. In a retrospective study done with a population of 87 patients with SCAD, eighty-seven percent of the patients were female and the mean age was 43 with an age range of 18 to 78 years old for both genders [8]. In addition, among those women with SCAD, $18 \%$ of them were noted to be postpartum. Predisposing factors to the development of SCAD include extreme physical activity, drugs, fibromuscular dysplasia, multiparity, connective tissue disorders, hormonal therapy and inflamma- tory conditions $[4-5,9]$.

\section{Pathophysiology}

SCAD can be atherosclerotic or non-atherosclerotic. Non-atherosclerotic SCAD is more common in the young population where the atheroma just starts to develop. The proposed mechanisms for the non-atherosclerotic SCAD is bleeding of the vasa vasorum or intimal tear causing intermedial hemorrhage [10]. Both of these result in the creation of false lumen [11]. Atherosclerotic SCAD on the other hand, is associated with plaque rupture causing disruption of the intimal-medial junction [1215]. In the two main types of SCAD, the expansion of the false lumen can subsequently cause ischemia and infarction.

In pregnant patients, the physiologic changes of pregnancy causing the hemodynamic stress and the possible hormonal effects on the arterial wall are considered to be the mechanisms for this group $[16,17]$.

\section{Clinical presentation and angiographic findings}

Clinical presentation may vary from mild chest pain to sudden 
cardiac death. Other presenting symptoms may include diaphoresis, dyspnea or vomiting $[5,8]$. However, female patients less than 50 years old with no traditional cardiovascular risk factors who suffered from ACS should raise the suspicion for SCAD [4].

In a retrospective study done among patients with SCAD in 2012, the most common presenting diagnosis was STEMI followed by NSTEMI. In some patients, there were note of ventricular fibrillation that were associated with STEMI [8]. Angiographically, the most commonly affected vessel is the left anterior descending artery but in some cases, multivessel disease was also noted [8].

\section{Treatment}

There are no treatment guidelines in the treatment of SCAD. Management may vary from medical therapy to emergency revascularization $[9,18]$. In most patients, after the diagnosis of SCAD has been established, conservative management is recommended [8]. However, the presence of ongoing or recurrent chest pain or ischemia on ECG, cardiogenic shock, unstable rhythms such as ventricular tachycardia or ventricular fibrillation, or involvement of the left main coronary artery, revascularization is necessary either via PCI or coronary artery bypass surgery [4]. These high risk features guide clinicians decide whether to do conservative or invasive management.

It is unclear if standard ACS agents are beneficial to SCAD patients. The role of antiplatelet in SCAD managed conservatively is unclear. However, since aspirin offers secondary prevention of CAD and with its low side effect profile and bleeding risk, it is considered reasonable to give aspirin for acute and long term SCAD treatment [4, 18].

Anticoagulation may aggravate and extend the dissection or it may dissolve any overlying thrombus improving lumen patency. Usual practice is that anticoagulants are discontinued after SCAD is confirmed through angiography to avoid extension of the dissection. Thrombolysis should be avoided due to reports of extension of dissection and clinical deterioration $[4,18]$. However, thrombolysis should not be withheld for patients with STEMI since the overall frequency of thrombotic occlusion is much higher than SCAD.

Coronary interventional procedures might themselves delay arterial wall healing or be harmful to injured vessels. In one study that compared outcomes of SCAD patients who underwent revascularization and conservative therapy, they noted that with regards to the original vessel with SCAD, there is more recurrence of dissection among vascularized patients than those managed conservatively [19]. In addition, two thirds of SCAD patients in the conservative therapy group experienced vessel wall healing during follow-up [4, 20]. These findings suggest that coronary interventional procedures might themselves delay arterial wall healing or be harmful to injured vessels. A conservative management strategy, except in patients undergoing revascularization for ongoing ischemia in a relatively extensive vascular territory or for those with high risk features, may be associated with a lower rate of SCAD recurrence $[4,9,19]$.

\section{Prognosis}

In earlier reported of cases of SCAD, there is high mortality rate of up to $80 \%$ [20]. Hence, prompt recognition and immediate treatment is of great importance. Higher mortality rates were associated if the left main coronary artery is involved or multivessel disease is affected [21]. However, prognosis is good for majority of patients where SCAD was promptly recognized and managed immediately. Full spontaneous healing was observed at 40 months, and a $37 \%$ rate of major adverse cardiovascular event in 5 years $[4,9,19]$.

\section{Conclusions}

The case presented is consistent with earlier reports of SCAD. It still remains to be a rare cause of STEMI. However, this should be considered and suspected among young individuals with ACS. It has a high mortality rate if not recognized and treated early. Establishment of diagnosis impacts management especially in the setting of STEMI. Conservative management is the preferred approach but in the presence of high risk features such as chest pain, ischemia on ECG, cardiogenic shock, or unstable rhythm, which some are present in the case discussed, revascularization is necessary and the preferred strategy.

\section{References}

1. Ward MJ, Kripalani S, Zhu Y, Storrow AB, Dittus RS, Harrell FE, Jr., Self WH. Incidence of emergency department visits for ST-elevation myocardial infarction in a recent six-year period in the United States. Am J Cardiol. 2015;115(2):167-170.

2. DeWood MA, Spores J, Notske R, Mouser LT, Burroughs R, Golden MS, Lang HT. Prevalence of total coronary occlusion during the early hours of transmural myocardial infarction. N Engl J Med. 1980;303(16):897-902.

3. Saw J, Aymong E, Mancini GB, Sedlak T, Starovoytov A, Ricci D. Nonatherosclerotic coronary artery disease in young women. Can J Cardiol. 2014;30(7):814-819.

4. Yip A, Saw J. Spontaneous coronary artery dissection-A review. Cardiovasc Diagn Ther. 2015;5(1):37-48.

5. Mortensen KH, Thuesen L, Kristensen IB, Christiansen EH. Spontaneous coronary artery dissection: a Western Denmark Heart Registry study. Catheter Cardiovasc Interv. 2009;74(5):710-717.

6. Nishiguchi T, Tanaka A, Ozaki Y, Taruya A, Fukuda S, Taguchi H, Iwaguro T, et al. Prevalence of spontaneous coronary artery dissection in patients with acute coronary syndrome. Eur Heart J Acute Cardiovasc Care. 2016;5(3):263-270.

7. McGill HC, Jr., McMahan CA, Zieske AW, Tracy RE, Malcom GT, Herderick EE, Strong JP. Association of coronary heart disease risk factors with microscopic qualities of coronary atherosclerosis in youth. Circulation. 2000;102(4):374-379. 
8. Tweet MS, Hayes SN, Pitta SR, Simari RD, Lerman A, Lennon RJ, Gersh BJ, et al. Clinical features, management, and prognosis of spontaneous coronary artery dissection. Circulation. 2012;126(5):579-588.

9. Saw J, Aymong E, Sedlak T, Buller CE, Starovoytov A, Ricci D, Robinson S, et al. Spontaneous coronary artery dissection: association with predisposing arteriopathies and precipitating stressors and cardiovascular outcomes. Circ Cardiovasc Interv. 2014;7(5):645-655.

10. Alfonso F. Spontaneous coronary artery dissection: new insights from the tip of the iceberg? Circulation. 2012;126(6):667-670.

11. Alfonso F, Bastante T. Spontaneous coronary artery dissection: novel diagnostic insights from large series of patients. Circ Cardiovasc Interv. 2014;7(5):638-641.

12. Almafragi A, Convens C, Heuvel PV. Spontaneous healing of spontaneous coronary artery dissection. Cardiol J. 2010;17(1):92-95.

13. Kansara P, Graham S. Spontaneous coronary artery dissection: case series with extended follow up. J Invasive Cardiol. 2011;23(2):76-80.

14. Shamloo BK, Chintala RS, Nasur A, Ghazvini M, Shariat P, Diggs JA, Singh SN. Spontaneous coronary artery dissection: aggressive vs. conservative therapy. J Invasive Cardiol. 2010;22(5):222-228.
15. Adlam D, Cuculi F, Lim C, Banning A. Management of spontaneous coronary artery dissection in the primary percutaneous coronary intervention era. J Invasive Cardiol. 2010;22(11):549-553.

16. Basso C, Morgagni GL, Thiene G. Spontaneous coronary artery dissection: a neglected cause of acute myocardial ischaemia and sudden death. Heart. 1996;75(5):451-454.

17. Vijayaraghavan R, Verma S, Gupta N, Saw J. Pregnancyrelated spontaneous coronary artery dissection. Circulation. 2014;130(21):1915-1920.

18. Alfonso F, Paulo M, Lennie V, Dutary J, Bernardo E, Jimenez-Quevedo P, Gonzalo N, et al. Spontaneous coronary artery dissection: long-term follow-up of a large series of patients prospectively managed with a "conservative" therapeutic strategy. JACC Cardiovasc Interv. 2012;5(10):1062-1070.

19. Dhawan R, Singh G, Fesniak H. Spontaneous coronary artery dissection: the clinical spectrum. Angiology. 2002;53(1):89-93.

20. Nakashima T, Noguchi T, Haruta S, Yamamoto Y, Oshima S, Nakao K, Taniguchi Y, et al. Prognostic impact of spontaneous coronary artery dissection in young female patients with acute myocardial infarction: A report from the Angina Pectoris-Myocardial Infarction Multicenter Investigators in Japan. Int J Cardiol. 2016;207:341-348. 